MATHEMATICS OF COMPUTATION

Volume 75, Number 256, October 2006, Pages 1697-1719

S 0025-5718(06)01884-9

Article electronically published on July 6, 2006

\title{
THE CONVERGENCE OF V-CYCLE MULTIGRID ALGORITHMS FOR AXISYMMETRIC LAPLACE AND MAXWELL EQUATIONS
}

\author{
JAYADEEP GOPALAKRISHNAN AND JOSEPH E. PASCIAK
}

\begin{abstract}
We investigate some simple finite element discretizations for the axisymmetric Laplace equation and the azimuthal component of the axisymmetric Maxwell equations as well as multigrid algorithms for these discretizations. Our analysis is targeted at simple model problems and our main result is that the standard V-cycle with point smoothing converges at a rate independent of the number of unknowns. This is contrary to suggestions in the existing literature that line relaxations and semicoarsening are needed in multigrid algorithms to overcome difficulties caused by the singularities in the axisymmetric Maxwell problems. Our multigrid analysis proceeds by applying the well known regularity based multigrid theory. In order to apply this theory, we prove regularity results for the axisymmetric Laplace and Maxwell equations in certain weighted Sobolev spaces. These, together with some new finite element error estimates in certain weighted Sobolev norms, are the main ingredients of our analysis.
\end{abstract}

\section{INTRODUCTION}

In this paper, we prove the optimality of the standard multigrid V-cycle applied to certain finite element discretizations of axisymmetric Laplace and Maxwell equations. In particular, it follows from our results that it is not necessary to do line smoothing or semicoarsening to obtain optimal multigrid convergence, in spite of the singular coefficients in the partial differential operators. This is contrary to suggestions in the existing literature that line relaxations and semicoarsening are needed in multigrid algorithms to overcome difficulties caused by the singularities in axisymmetric problems.

In the presence of axisymmetry, the three dimensional Laplace equation $-\Delta u=$ $f$ reduces to the two dimensional partial differential equation

$$
-\frac{1}{r} \frac{\partial}{\partial r}\left(r \frac{\partial u}{\partial r}\right)-\frac{\partial^{2} u}{\partial z^{2}}=f, \quad \text { on } D .
$$

Received by the editor May 20, 2004 and, in revised form, September 16, 2005.

2000 Mathematics Subject Classification. Primary 65F10, 65M55, 65N55, 65N30, 49N60, 74G15, 35Q60.

Key words and phrases. Multigrid, axisymmetric, Laplace equation, Maxwell equations, Vcycle, Jacobi, Gauss-Seidel, regularity, bilinear, finite element.

This work was supported in part by NSF grant numbers DMS-0410030 and DMS-0311902. We also gratefully acknowledge support from ICES, The University of Texas at Austin.

(C)2006 American Mathematical Society Reverts to public domain 28 years from publication 
For simplicity, we shall only consider the case when the domain $D$ is the unit square. Note that the $\{r=0\}$ line intersects the boundary $\partial D$, so the coefficients in (1.1) are truly singular. Herein lies the difficulty in analysis of this problem. While the reduction from three to two space dimensions obviously results in substantial computational savings, the introduction of singular coefficients in the differential operator was thought to be a problem, at least in the analysis of iterative solution by multigrid methods. But as we shall show, a standard multigrid V-cycle with point smoothing converges uniformly at a rate independent of the number of unknowns.

When using multigrid algorithms for second order problems that are not uniformly elliptic, it is well known that, in many cases, one needs to perform line smoothing to obtain optimal algorithms [4, 8, 9, 10, 14, 15. A rigorous proof of the optimality of the V-cycle with line smoothing for a discretization of the operator

$$
-\frac{\partial}{\partial x}\left(a(x, y) \frac{\partial}{\partial x}\right)-\frac{\partial}{\partial y}\left(b(x, y) \frac{\partial}{\partial y}\right)
$$

when $a(x, y)>0$ is of unit size and $b(x, y)>0$ is allowed to be arbitrarily close to zero, is given in 8, 14. Clearly, the partial differential operator in (1.1) is not a specific instance of the operator considered in [8, although there are some similarities. Our contention is that although line relaxation may be necessary to obtain uniform multigrid convergence for (1.2) with degenerate $b$, it is not necessary for the operator in (1.1).

The paper [5] considers equations of the form (1.2) where the coefficients $a$ and $b$ are given in tensor product form and have different types of singularities near $x=0$. There they suggest line smoothing and semicoarsening. While this may be necessary for some examples considered in [5], as we shall see, neither is necessary for (1.1).

We also study the case of axisymmetric Maxwell equations. In the presence of axisymmetry, it is well known that three dimensional vector Maxwell equations decouple into two systems of equations, one for the azimuthal component and another for the meridian components [2, 6. The equation for the azimuthal component is

$$
-\frac{\partial}{\partial r}\left(\frac{1}{r} \frac{\partial}{\partial r}(r u)\right)-\frac{\partial^{2} u}{\partial z^{2}}=f .
$$

This scalar equation can be analyzed by techniques somewhat similar to the axisymmetric Laplace equation (1.1). We will investigate two distinct finite element discretizations for this equation, one using bilinear elements and another using a finite element space of linear combinations of $r, 1 / r, r z$, and $z / r$. Both discretizations yield systems for which we can prove that the multigrid V-cycle converges uniformly at a rate independent of the mesh size. Multigrid algorithms for the azimuthal Maxwell equation (1.3) have been studied previously in 6]. However, the algorithm considered there resorts to line smoothing as well as semicoarsening. As in the case of the axisymmetric Laplace equation, neither is necessary.

While spectral discretizations of certain axisymmetric problems have been thoroughly studied [3], finite element approximations seem to have been less studied. Therefore, before proceeding to multigrid analyses, we introduce the weak formulations of our problems in certain weighted Sobolev spaces and prove new finite element approximation estimates. In particular, our estimates bound the finite element error in weighted Sobolev spaces using norms of the data that are appropriate for multigrid analyses. Our multigrid analysis proceeds by verifying the well known 
conditions of the regularity based multigrid theory. In order to apply this theory, we prove regularity results for the axisymmetric Laplace and Maxwell equations in certain weighted Sobolev spaces. While most of our regularity estimates are consequences of the well known regularity results for the three dimensional problems, one (Theorem 4.1) appears to be peculiar to the axisymmetric case.

Weighted Sobolev spaces have been used to obtain optimal multigrid convergence estimates. For example, [17] used weighted Sobolev spaces to analyze the behavior of multigrid methods when applied to problems with point singularities such as those resulting from second order problems with re-entrant corners.

The outline of the remainder of the paper is as follows. We first introduce the weak setting for the axisymmetric Laplace problem and prove finite element estimates (Section 2). In Section 3, we introduce the weak formulation of the azimuthal Maxwell problem, prove a regularity estimate, and prove finite element convergence. In Section 4, we continue our investigation into azimuthal Maxwell problem by proving convergence of a different finite element discretization. The multigrid convergence analysis is given in Section 5 . Numerical experiments illustrating the theoretical results are given in Section 6. Finally, proofs of technical lemmas are gathered in Appendix $\mathrm{A}$.

\section{The AXISymmetric Laplace EQUATion}

In this section, we consider the axisymmetric Laplace equation on the unit square $D$ in the $r-z$ plane (so the $\{r=0\}$ line intersects $\partial D$ ). Let $\Gamma_{0}$ be the part of $\partial D$ along the $z$-axis and $\Gamma_{1}$ be the remainder of $\partial D$. We then formally have the partial differential equation

$$
\begin{aligned}
\mathcal{L} u=f & \text { on } D, \\
u=0 & \text { on } \Gamma_{1},
\end{aligned}
$$

where

$$
\mathcal{L}=-\frac{1}{r} \frac{\partial}{\partial r}\left(r \frac{\partial}{\partial r}\right)-\frac{\partial^{2}}{\partial z^{2}}
$$

If the solution is smooth, the axial symmetry implies the boundary condition $\partial_{r} u=0$ on $\Gamma_{0}$. A weaker natural boundary condition is imposed in a subsequent variational formulation. We shall approximate this problem using bilinear finite elements. Note that more general domains can be handled, e.g., by employing standard linear triangular elements away from $\Gamma_{0}$ and bilinear elements near $\Gamma_{0}$. Then the analysis can proceed by combining the new estimates we shall develop near $\Gamma_{0}$ with the standard finite element estimates for triangular elements. We omit such generalizations in the interest of simplicity.

2.1. The weak solution. The variational formulation that we consider is selected so that its solutions coincide with the meridian trace of the axisymmetric solutions of the weak form of the three dimensional Laplace equation (2.1). Let $L_{r}^{2}(D)$ denote the weighted Lebesgue space of all measurable functions on $D$ for which $\|u\|_{L_{r}^{2}(D)} \equiv \int_{D} u^{2} r \mathrm{~d} r \mathrm{~d} z<\infty$. The weighted Sobolev space $H_{r}^{k}(D)$ consists of all functions in $L_{r}^{2}(D)$ whose distributional derivatives of order $k$ are also in $L_{r}^{2}(D)$. 
Sobolev seminorms and norms are denoted in the standard way, e.g.,

$$
\begin{aligned}
& |v|_{H_{r}^{1}(D)}^{2}=\int_{D}\left(\left|\partial_{r} v\right|^{2}+\left|\partial_{z} v\right|^{2}\right) r \mathrm{~d} r \mathrm{~d} z \\
& |v|_{H_{r}^{2}(D)}^{2}=\int_{D}\left(\left|\partial_{r r} v\right|^{2}+\left|\partial_{r z} v\right|^{2}+\left|\partial_{z z} v\right|^{2}\right) r \mathrm{~d} r \mathrm{~d} z .
\end{aligned}
$$

The following properties of these weighted spaces will be useful.

Proposition 2.1. (1) $C^{\infty}(\bar{D})$ is dense in $H_{r}^{k}(D)$.

(2) For all $v \in H_{r}^{1}(D)$, on any horizontal edge $E_{a}=\{(r, a) \in D: 0<r<1\}$,

$$
\frac{1}{2} \int_{E_{a}} r v^{2} \mathrm{~d} r \leq\|v\|_{L_{r}^{2}(D)}^{2}+\left\|\partial_{z} v\right\|_{L_{r}^{2}(D)}^{2}
$$

(3) For all $v \in H_{r}^{1}(D)$ with $\left.v\right|_{E_{a}}=0$ for some $0<a<1$,

$$
\|v\|_{L_{r}^{2}(D)} \leq\left\|\partial_{z} v\right\|_{L_{r}^{2}(D)} .
$$

The first assertion of the proposition is a well known fact (see, e.g., 13, Theorem 11.2]). It is easy to verify (2.2) for all $v \in C^{\infty}(\bar{D})$, so the result follows by the density asserted in the first part. Note that it follows from Proposition 2.1(2) that traces exist on $z=0$ and $z=1$ edges of $\partial D$ (in a one dimensional weighted $L^{2}$ space). Traces obviously exist on the $r=1$ edge, as in a neighborhood of that edge the function is actually in a standard Sobolev space. Note that traces of $H_{r}^{1}(D)$ functions do not exist, in general, on the $r=0$ edge of $\partial D$. The last inequality of Proposition 2.1 is a Poincaré inequality which also can be easily established using the density of smooth functions.

Now we introduce the first variational problem that we shall study. Define

$$
\begin{aligned}
a_{r}(v, w) & =\int_{D} r\left(\partial_{r} v \partial_{r} w+\partial_{z} v \partial_{z} w\right) \mathrm{d} r \mathrm{~d} z, \\
(v, w)_{r} & =\int_{D} r v w \mathrm{~d} r \mathrm{~d} z, \quad \text { and } \\
V & =\left\{v \in H_{r}^{1}(D):\left.v\right|_{\Gamma_{1}}=0\right\} .
\end{aligned}
$$

By Proposition 2.1(2), $V$ is well defined. We are interested in approximating $u \in V$ satisfying

$$
a_{r}(u, v)=(f, v)_{r}, \quad \text { for all } v \in V
$$

for some $f \in L_{r}^{2}(D)$. By Proposition 2.1(13), the conditions of the Lax-Milgram lemma are verified so there is a unique weak solution $u$ in $V$.

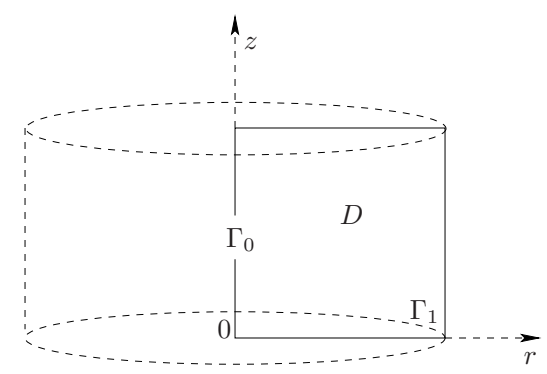

Figure 1. The computational domain and its revolution 
As is well known, the weak solution of (2.4) is related to the solution of a three dimensional axisymmetric Dirichlet problem. Let $\Omega=\{(r, \theta, z): 0 \leq r<1,0<$ $z<1,0 \leq \theta<2 \pi\}$, where $(r, \theta, z)$ are cylindrical coordinates (so $\Omega$ is the revolution of $D$ about the $z$-axis; see Figure 11). We denote the subspace of axisymmetric elements of $H^{k}(\Omega)$ by $\breve{H}^{k}(\Omega)$ and $\breve{L}^{2}(\Omega) \equiv \breve{H}^{0}(\Omega)$ (for definitions of axisymmetric Sobolev spaces see [3]). The restriction map $g(r, \theta, z) \mapsto g_{D}(r, z)$ given by

$$
g_{D}(r, z)=g(r, 0, z), \quad \text { for all }(r, z) \in D,
$$

is well defined for smooth functions and extends to an isometry from $\breve{L}^{2}(\Omega)$ onto $L_{r}^{2}(D)$ :

$$
2 \pi\left\|g_{D}\right\|_{L_{r}^{2}(D)}^{2}=\|g\|_{L^{2}(\Omega)}^{2} .
$$

The action of the inverse of the restriction map $g \mapsto g_{D}$ will be denoted by superscripting functions with $\Omega$, e.g., $\left(g_{D}\right)^{\Omega}=g$. Then, if $U$ is the solution of the three dimensional Dirichlet problem

$$
-\Delta U=f^{\Omega} \quad \text { on } \Omega, \quad U=0 \quad \text { on } \partial \Omega,
$$

it is easy to see that [3, Proposition II.4.1]

$$
u^{\Omega}=U,
$$

where $u$ solves (2.4). The following regularity estimate will be useful in our subsequent multigrid analysis.

Theorem 2.1. There is a constant $C_{\text {reg }}$ such that for all $f \in L_{r}^{2}(D)$ the solution $u$ of (2.4) satisfies

$$
|u|_{H_{r}^{2}(D)} \leq C_{\mathrm{reg}}\|f\|_{L_{r}^{2}(D)} .
$$

Proof. Since $\Omega$ is convex, by a standard regularity result, the solution $U$ of (2.7) is in $H^{2}(\Omega)$ and there is a constant $C_{\text {reg }}$ such that

$$
\|U\|_{H^{2}(\Omega)} \leq C_{\mathrm{reg}}\left\|f^{\Omega}\right\|_{L^{2}(\Omega)} .
$$

Thus,

$$
\begin{aligned}
|U|_{H^{2}(\Omega)}^{2}= & \int_{\Omega}\left(\left|\partial_{x}^{2} U\right|^{2}+\left|\partial_{y}^{2} U\right|^{2}+\left|\partial_{z}^{2} U\right|^{2}\right. \\
& \left.\quad+2\left|\partial_{x} \partial_{y} U\right|^{2}+2\left|\partial_{y} \partial_{z} U\right|^{2}+2\left|\partial_{z} \partial_{x} U\right|^{2}\right) \mathrm{d} x \mathrm{~d} y \mathrm{~d} z \\
= & \int_{0}^{2 \pi} \int_{0}^{1} \int_{0}^{1} r\left(\left|\partial_{z}^{2} U\right|^{2}+\left|\partial_{r}^{2} U\right|^{2}+2\left|\partial_{r} \partial_{z} U\right|^{2}+\left|\frac{\partial_{r} U}{r}\right|^{2}\right) \mathrm{d} r \mathrm{~d} z \mathrm{~d} \theta \\
= & 2 \pi \int_{D} r\left(\left|\partial_{z}^{2} u\right|^{2}+\left|\partial_{r}^{2} u\right|^{2}+2\left|\partial_{r} \partial_{z} u\right|^{2}+\left|\frac{\partial_{r} u}{r}\right|^{2}\right) \mathrm{d} r \mathrm{~d} z \\
\geq & 2 \pi|u|_{H_{r}^{2}(D)}^{2},
\end{aligned}
$$

and the result follows by (2.6), (2.8), and (2.9).

2.2. An approximation estimate. We use bilinear finite elements to discretize (2.4). Let the domain $D=(0,1)^{2}$ be partitioned into squares with vertices $(i h, j h)$, $h=1 / n$. This forms the mesh $\mathcal{T}_{h}$. Let $V_{h} \subseteq V$ denote the standard bilinear finite element space with respect to mesh $\mathcal{T}_{h}$. The finite element approximation of $u$ is the function $u_{h} \in V_{h}$ satisfying

$$
a_{r}\left(u_{h}, v_{h}\right)=\left(f, v_{h}\right)_{r} \quad \text { for all } v_{h} \in V_{h} .
$$


To obtain an error estimate, as well as for multigrid analysis later, we need to construct an interpolation operator with approximation properties in the weighted norms. Define $\Pi_{h}: H_{r}^{2}(\Omega) \mapsto V_{h}$ element by element as follows. On every element $K$ whose boundary does not intersect $\Gamma_{0},\left.\left(\Pi_{h} v\right)\right|_{K}$ is the unique bilinear function whose value at the four vertices of $K$ coincides with that of $v$. The remaining elements are of the form $\left(0, r_{1}\right) \times\left(z_{0}, z_{1}\right)$. On such an element $K,\left.\left(\Pi_{h} v\right)\right|_{K}$ is defined to be the unique bilinear function satisfying

$$
\begin{aligned}
\left(\Pi_{h} v\right)\left(r_{1}, z_{0}\right) & =v\left(r_{1}, z_{0}\right), \\
\left(\Pi_{h} v\right)\left(r_{1}, z_{1}\right) & =v\left(r_{1}, z_{1}\right), \\
\int_{0}^{r_{1}} \rho^{1 / 2}\left(\partial_{r} \Pi_{h} v\right)\left(\rho, z_{0}\right) \mathrm{d} \rho & =\int_{0}^{r_{1}} \rho^{1 / 2} \partial_{r} v\left(\rho, z_{0}\right) \mathrm{d} \rho \\
\int_{0}^{r_{1}} \rho^{1 / 2}\left(\partial_{r} \Pi_{h} v\right)\left(\rho, z_{1}\right) \mathrm{d} \rho & =\int_{0}^{r_{1}} \rho^{1 / 2} \partial_{r} v\left(\rho, z_{1}\right) \mathrm{d} \rho .
\end{aligned}
$$

Note that $\Pi_{h} v$ is defined for all $v \in H_{r}^{2}(D)$ because each of the right hand sides above define a continuous functional in $H_{r}^{2}(D)$; for example, the functional $v \mapsto \int_{0}^{r_{1}} \rho^{1 / 2} \partial_{r} v\left(\rho, z_{0}\right) \mathrm{d} \rho$, is continuous on all $H_{r}^{2}(D)$ by Proposition 2.1 (2), while the functional $v \mapsto v\left(r_{1}, z_{0}\right)$ is continuous because a Sobolev inequality holds away from $r=0$. It is also easily seen that the linear system defining $\Pi_{h}$ on each element is uniquely solvable and that $\Pi_{h} v$ is a continuous function on $D$. The change in the definition of $\Pi_{h} v$ on elements with an edge on the $r=0$ axis is necessitated by the fact that $v \mapsto v\left(0, z_{i}\right)$ is not a continuous functional on $H_{r}^{2}(D)$.

We prove an approximation estimate for $\Pi_{h}$ using a few intermediate lemmas. In these lemmas, $K \subseteq D$ is the square given by

$$
K=\left\{(r, z): r_{0}<r<r_{1}, z_{0}<z<z_{1}\right\},
$$

where $z_{1}=z_{0}+h$ and $r_{1}=r_{0}+h$. Proofs of all lemmas are in Appendix A

Lemma 2.1. Let $v \in H_{r}^{1}(K)$ satisfy any one of the following conditions:

$$
\begin{aligned}
& \int_{z_{0}}^{z_{1}} v\left(r_{1}, \zeta\right) \mathrm{d} \zeta=0 . \\
& \int_{r_{0}}^{r_{1}} v\left(\rho, z_{0}\right) \mathrm{d} \rho=0 \text { and } r_{0}>0 . \\
& \int_{r_{0}}^{r_{1}} \rho^{1 / 2} v\left(\rho, z_{0}\right) \mathrm{d} \rho=0 \text { and } r_{0}=0 . \\
& \int_{r_{0}}^{r_{1}} \rho v\left(\rho, z_{0}\right) \mathrm{d} \rho=0 .
\end{aligned}
$$

Then

$$
\|v\|_{L_{r}^{2}(K)}^{2} \leq 3 h^{2}|v|_{H_{r}^{1}(K)}^{2}
$$

Lemma 2.2. For all $v \in H_{r}^{2}(K)$,

$$
\left\|\partial_{r z}\left(\Pi_{h} v\right)\right\|_{L_{r}^{2}(K)}^{2} \leq \frac{3}{2}\left\|\partial_{r z} v\right\|_{L_{r}^{2}(K)}^{2} .
$$

Theorem 2.2. For all $v \in H_{r}^{2}(D)$,

$$
\left|v-\Pi_{h} v\right|_{H_{r}^{1}(D)} \leq \sqrt{15} h|v|_{H_{r}^{2}(D)} .
$$


As a consequence, the discrete solution $u_{h}$ approximates the exact solution u of (2.4):

$$
\left|u-u_{h}\right|_{H_{r}^{1}(D)} \leq C h\|f\|_{L_{r}^{2}(D)} .
$$

Proof. Since $\partial_{z}\left(v-\Pi_{h} v\right)$ satisfies (2.13) and $\partial_{r}\left(v-\Pi_{h} v\right)$ satisfies (2.14) or (2.15), by Lemma 2.1 we have

$$
\begin{aligned}
\left|v-\Pi_{h} v\right|_{H_{r}^{1}(K)}^{2} \leq & 3 h^{2}\left(\left\|\partial_{r r}\left(u-\Pi_{h} u\right)\right\|_{L_{r}^{2}(K)}^{2}+\left\|\partial_{r z}\left(u-\Pi_{h} u\right)\right\|_{L_{r}^{2}(K)}^{2}\right) \\
& +3 h^{2}\left(\left\|\partial_{r z}\left(u-\Pi_{h} u\right)\right\|_{L_{r}^{2}(K)}^{2}+\left\|\partial_{z z}\left(u-\Pi_{h} u\right)\right\|_{L_{r}^{2}(K)}^{2}\right) .
\end{aligned}
$$

Since $\partial_{r r}\left(\Pi_{h} v\right)=\partial_{z z}\left(\Pi_{h} v\right)=0$, this implies

$$
\left|v-\Pi_{h} v\right|_{H_{r}^{1}(K)}^{2} \leq 3 h^{2}\left\|\partial_{r r} v\right\|_{L_{r}^{2}(K)}^{2}+3 h^{2}\left\|\partial_{z z} v\right\|_{L_{r}^{2}(K)}^{2}+6 h^{2}\left\|\partial_{r z}\left(v-\Pi_{h} v\right)\right\|_{L_{r}^{2}(K)}^{2} .
$$

By Lemma 2.2.

$$
\begin{aligned}
\left\|\partial_{r z}\left(v-\Pi_{h} v\right)\right\|_{L_{r}^{2}(K)}^{2} & \leq 2\left\|\partial_{r z} v\right\|_{L_{r}^{2}(K)}^{2}+2\left\|\partial_{r z}\left(\Pi_{h} v\right)\right\|_{L_{r}^{2}(K)}^{2} \\
& \leq 5\left\|\partial_{r z} v\right\|_{L_{r}^{2}(K)}^{2} .
\end{aligned}
$$

The interpolation error estimate of the theorem follows by using this estimate in (2.17). The second estimate of the theorem follows from the first and the regularity estimate of Theorem 2.1.

\section{The azimuthal Maxwell problem}

In this section, we shall study a finite element discretization for the azimuthal component of the axisymmetric Maxwell equations. Formally, the governing partial differential equation is

$$
\begin{aligned}
\mathcal{L}_{\theta} u=f & \text { on } D, \\
u=0 & \text { on } \partial D,
\end{aligned}
$$

where

$$
\mathcal{L}_{\theta} v=-\frac{\partial}{\partial r}\left(\frac{1}{r} \frac{\partial}{\partial r}(r v)\right)-\frac{\partial^{2} v}{\partial z^{2}} .
$$

We investigate two approaches to a finite element discretization of this equation, one using standard bilinear elements, and another using a finite element space built using linear combinations of $r, 1 / r, r z$, and $z / r$. In this section, we will investigate the latter. The next section is devoted to the former. Just as linear functions are in the kernel of the standard Laplace operator $\partial_{r r}+\partial_{z z}$, the span of $r, 1 / r, r z$, and $z / r$ is in the kernel of $\mathcal{L}_{\theta}$. Hence, considering finite elements built using the span of $r, 1 / r, r z$, and $z / r$ is as natural as considering linear finite elements for the Laplace equation. We begin by specifying the weak problem and associated spaces.

3.1. A weak formulation. To give a variational formulation of (3.1) together with appropriate boundary conditions, we start with an appropriate Sobolev space. Let $\widetilde{H}_{r}^{1}(D)$ denote $H_{r}^{1}(D) \cap L_{1 / r}^{2}(D)$ where $L_{1 / r}^{2}(D)$ is the set of all measurable $v$ for which

$$
\|v\|_{L_{1 / r}^{2}(D)}^{2} \equiv \int_{D} r^{-1} v^{2} \mathrm{~d} r \mathrm{~d} z<\infty .
$$

$\widetilde{H}_{r}^{1}(D)$ is a Hilbert space with the norm

$$
\|v\|_{\widetilde{H}_{r}^{1}(D)}=\left(\|v\|_{H_{r}^{1}(D)}^{2}+\|v\|_{L_{1 / r}^{2}(D)}^{2}\right)^{1 / 2} .
$$


Lemma 3.1. The set of $C^{\infty}(\bar{D})$ functions which vanish in a neighborhood of $\Gamma_{0}$ (the $\{r=0\}$ line segment) is dense in $\widetilde{H}_{r}^{1}(D)$.

As before, proofs of all lemmas are in Appendix A. In order to clarify the nature of traces of $\widetilde{H}_{r}^{1}(D)$ as well as for later arguments, the following lemma will be useful.

Lemma 3.2. Let $K$ be a square contained in $D$ with vertices as in (2.12). For all $v \in \widetilde{H}_{r}^{1}(K)$, the trace of $v$ on a vertical line segment $S_{a}=\left\{(a, z) \in K: z_{0}<z<z_{1}\right\}$ exists for any $a \in\left[r_{0}, r_{1}\right]$ and

$$
\|v\|_{L^{2}\left(S_{a}\right)}^{2} \leq\left\|\partial_{r} v\right\|_{L_{r}^{2}(K)}^{2}+\frac{r_{1}+h}{h}\|v\|_{L_{1 / r}^{2}(K)} .
$$

The lemma shows that unlike $H_{r}^{1}(D)$, functions in $\widetilde{H}_{r}^{1}(D)$ have traces in $L^{2}$ even on the $r=0$ edge. Moreover, since functions in $C^{\infty}(\bar{D})$ which vanish in a neighborhood of $\Gamma_{0}$ have zero traces on this edge, by the density given by Lemma 3.1. traces of all functions in $\widetilde{H}_{r}^{1}(D)$ must vanish on that edge. Traces on the remainder of $\partial D$ obviously exist by Proposition 2.1.

Proposition 3.1. For all $v \in \widetilde{H}_{r}^{1}(D), \partial_{r}(r v)$ is in $L_{1 / r}^{2}(D)$ and satisfies

$$
\left\|\partial_{r} v\right\|_{L_{r}^{2}(D)}^{2}+\|v\|_{L_{1 / r}^{2}(D)}^{2} \leq\left\|\partial_{r}(r v)\right\|_{L_{1 / r}^{2}(D)}^{2} \leq 2\left\|\partial_{r} v\right\|_{L_{r}^{2}(D)}^{2}+2\|v\|_{L_{1 / r}^{2}(D)}^{2} .
$$

Proof. The upper inequality is obvious for smooth functions, so by Lemma 3.1 , $\partial_{r}(r v)$ exists in $L_{1 / r}^{2}(D)$ (and satisfies the same inequality).

For the other direction, note that if $v \in C^{\infty}(\bar{D})$ and vanishes in a neighborhood of $\Gamma_{0}$,

$$
\int_{D} 2 v \partial_{r} v \mathrm{~d} r \mathrm{~d} z=\int_{0}^{1} \int_{0}^{1} \partial_{r}\left(v^{2}\right) \mathrm{d} r \mathrm{~d} z=\int_{0}^{1} v^{2}(1, z) \mathrm{d} z \geq 0
$$

Hence,

$$
\begin{aligned}
\int_{D} r\left|\frac{\partial_{r}(r v)}{r}\right|^{2} \mathrm{~d} r \mathrm{~d} z & =\int_{D} r\left(\left|\partial_{r} v\right|^{2}+\frac{v^{2}}{r^{2}}+2 \frac{v}{r} \partial_{r} v\right) \mathrm{d} r \mathrm{~d} z \\
& \geq \int_{D} r\left(\left|\partial_{r} v\right|^{2}+\frac{v^{2}}{r^{2}}\right) \mathrm{d} r \mathrm{~d} z .
\end{aligned}
$$

Using Lemma 3.1, we get the lower inequality for all $v \in \widetilde{H}_{r}^{1}(D)$.

Now we can state a well posed weak problem for the azimuthal Maxwell equation with electric boundary conditions. Defining

$$
V^{\theta}=\left\{v \in \widetilde{H}_{r}^{1}(D): v=0 \text { on } \partial D\right\},
$$

the weak problem can be stated as follows for any given $f \in L_{r}^{2}(D)$. Find $u \in V^{\theta}$ satisfying

$$
a_{\theta}(u, v)=(f, v)_{r} \quad \text { for all } v \in V^{\theta}
$$

where

$$
a_{\theta}(v, w)=\int_{D} \frac{1}{r} \partial_{r}(r v) \partial_{r}(r w) \mathrm{d} r \mathrm{~d} z+\int_{D} r\left(\partial_{z} v\right)\left(\partial_{z} w\right) \mathrm{d} r \mathrm{~d} z .
$$

This bilinear form is continuous by Proposition 3.1 and coercive by Proposition 2.1(3). Hence, by the Lax-Milgram lemma, there is a unique $u \in V^{\theta}$ satisfying (3.3). 
Now we give a regularity result for the weak solution. Let

$$
\begin{aligned}
|v|_{\widetilde{H}_{r}^{2}(D)}^{2} & =\left|r^{-1} \partial_{r}(r v)\right|_{H_{r}^{1}(D)}^{2}+\left|\partial_{z} v\right|_{H_{r}^{1}(D)}^{2}, \\
\|v\|_{\widetilde{H}_{r}^{2}(D)}^{2} & =|v|_{\widetilde{H}_{r}^{2}(D)}^{2}+\|v\|_{\widetilde{H}_{r}^{1}(D)}^{2}+\left\|\partial_{z} v\right\|_{L_{1 / r}^{2}(D)}^{2}, \\
\widetilde{H}_{r}^{2}(D) & =\left\{v \in \widetilde{H}_{r}^{1}(D):\|v\|_{\widetilde{H}_{r}^{2}(D)}^{2}<\infty\right\} .
\end{aligned}
$$

The following regularity theorem is a straightforward consequence of the well known regularity estimates for static Maxwell equations in three dimensions. It will be useful to recall that if $\boldsymbol{v}=v_{r} \boldsymbol{e}_{r}+v_{\theta} \boldsymbol{e}_{\theta}+v_{z} \boldsymbol{e}_{z}$ in cylindrical coordinates, then

$$
\operatorname{curl} \boldsymbol{v}=\left(\frac{1}{r} \frac{\partial v_{z}}{\partial \theta}-\frac{\partial v_{\theta}}{\partial z}\right) \boldsymbol{e}_{r}+\left(\frac{\partial v_{r}}{\partial z}-\frac{\partial v_{z}}{\partial r}\right) \boldsymbol{e}_{\theta}+\frac{1}{r}\left(\frac{\partial}{\partial r}\left(r v_{\theta}\right)-\frac{\partial v_{r}}{\partial \theta}\right) \boldsymbol{e}_{z} .
$$

Here $\boldsymbol{e}_{r}, \boldsymbol{e}_{\theta}$, and $\boldsymbol{e}_{z}$ denote unit vectors in the $r, \theta$, and $z$ directions, respectively.

Theorem 3.1. If $u$ solves (3.3) for an $f \in L_{r}^{2}(D)$, then $u \in \widetilde{H}_{r}^{2}(D)$ and

$$
\|u\|_{\widetilde{H}_{r}^{2}(D)}^{2} \leq C\|f\|_{L_{r}^{2}(D)}^{2} .
$$

Proof. Let $\mathcal{D}(D)$ denote the space of compactly supported infinitely differentiable functions on $D$. If $u$ solves (3.3) with an $f \in L_{r}^{2}(D)$, then the distribution $\mathcal{L}_{\theta} u$ satisfies

$$
\left(\mathcal{L}_{\theta} u, r \phi\right)=a_{\theta}(u, \phi)=(f, r \phi)
$$

for all $\phi \in \mathcal{D}(D)$. Since $\mathcal{D}(D)$ is dense in $L_{r}^{2}(D)$, this implies that $\mathcal{L}_{\theta} u$ and $f$ coincide as functions in $L_{r}^{2}(D)$. Now note that $\left(\mathcal{L}_{\theta} u\right)^{\Omega}$ is the $\theta$-component of curl curl $\boldsymbol{u}_{\theta}$ where $\boldsymbol{u}_{\theta}=u^{\Omega} \boldsymbol{e}_{\theta}$. Since the equality $\mathcal{L}_{\theta} u=f$ holds in $L_{r}^{2}(D)$, a corresponding identity holds in the isometric space $\breve{L}^{2}(\Omega)$ :

$$
\operatorname{curl} \operatorname{curl} \boldsymbol{u}_{\theta}=f^{\Omega} \boldsymbol{e}_{\theta} .
$$

Moreover,

$$
\begin{aligned}
\operatorname{div} \boldsymbol{u}_{\theta}=0 & \text { on } \Omega, \\
\boldsymbol{u}_{\theta} \times \boldsymbol{n}=0 & \text { on } \partial \Omega .
\end{aligned}
$$

Hence, by standard regularity estimates for vector potentials on convex domains [1],

$$
\left\|\operatorname{curl} \boldsymbol{u}_{\theta}\right\|_{\boldsymbol{H}^{1}(\Omega)}^{2}+\left\|\boldsymbol{u}_{\theta}\right\|_{\boldsymbol{H}^{1}(\Omega)}^{2} \leq C\|f\|_{L_{r}^{2}(\Omega)}^{2} .
$$

By (3.5), $\operatorname{curl} \boldsymbol{u}_{\theta}=-\left(\partial_{z} u\right) \boldsymbol{e}_{r}+r^{-1} \partial_{r}(r u) \boldsymbol{e}_{z}$. Since the $r, \theta$, and $z$ components of $\boldsymbol{H}^{1}(\Omega)$ are isometrically equivalent to $\widetilde{H}_{r}^{1}(D), \widetilde{H}_{r}^{1}(D)$, and $H_{r}^{1}(D)$, respectively [2, Proposition 3.17],

$$
\begin{aligned}
\frac{1}{2 \pi}\left(\left\|\operatorname{curl} \boldsymbol{u}_{\theta}\right\|_{\boldsymbol{H}^{1}(\Omega)}^{2}\right. & \left.+\left\|\boldsymbol{u}_{\theta}\right\|_{\boldsymbol{H}^{1}(\Omega)}^{2}\right) \\
& =\left\|\partial_{z} u\right\|_{\widetilde{H}_{r}^{1}(D)}^{2}+\left\|\frac{1}{r} \partial_{r}(r u)\right\|_{H_{r}^{1}(D)}^{2}+\|u\|_{\widetilde{H}_{r}^{1}(D)}^{2} \geq\|u\|_{\widetilde{H}_{r}^{2}(D)}^{2} .
\end{aligned}
$$

This completes the proof of the theorem. 
3.2. A finite element space. As mentioned earlier, we will consider two suitable finite element spaces for discretizing the weak problem, one in this section, and another in the next. The space we consider in this section is

$$
\widetilde{V}_{h}^{\theta}=\left\{v \in V^{\theta}:\left.v\right|_{K} \text { is in the span of } r, 1 / r, r z \text {, and } z / r \text { for all } K \in \mathcal{T}_{h}\right\},
$$

where, as before, $\mathcal{T}_{h}$ is the mesh of square elements that partition $D$. Note that locally constant functions are not in this space, yet we shall prove approximation properties by direct arguments. The finite element approximation is defined as the unique function $u_{h} \in \widetilde{V}_{h}^{\theta}$ satisfying

$$
a_{\theta}\left(u_{h}, v_{h}\right)=\left(f, v_{h}\right)_{r}, \quad \text { for all } v_{h} \in \widetilde{V}_{h}^{\theta} .
$$

To prove that $u_{h}$ approximates $u$, we first prove an approximation property of $\widetilde{V}_{h}^{\theta}$ using a nodal interpolation operator

$$
\widetilde{\Pi}_{h}^{\theta}: \widetilde{H}_{r}^{2}(D) \mapsto \widetilde{V}_{h}^{\theta} .
$$

On every element $K,\left.\left(\widetilde{\Pi}_{h}^{\theta} v\right)\right|_{K}$ is defined as the unique function in the span of $r, 1 / r, z$, and $z / r$ satisfying

$$
\left(\widetilde{\Pi}_{h}^{\theta} v\right)\left(r_{i}, z_{j}\right)=v\left(r_{i}, z_{j}\right), \quad i, j=0,1,
$$

where the notation for coordinates of vertices of $K$ is as in (2.12). The following proposition shows that $\widetilde{\Pi}_{h}^{\theta}$ is well defined on all functions in $\widetilde{H}_{r}^{2}(D)$.

Proposition 3.2. For all $w \in \widetilde{H}_{r}^{2}(D)$ and any point $(r, z) \in D$,

$$
|w(r, z)| \leq C\|w\|_{\widetilde{H}_{r}^{2}(D)} \cdot
$$

Proof. For any vertical edge $E$ containing $(r, z)$, we apply Lemma 3.2 with $K=D$ and $v=\partial_{z} w$ to get that

$$
\left\|\partial_{z} w\right\|_{L^{2}(E)}^{2} \leq C\left(\left\|\partial_{z} w\right\|_{L_{1 / r}^{2}(D)}^{2}+\left\|\partial_{r z} w\right\|_{L_{r}^{2}(D)}^{2}\right) \leq C\|w\|_{\widetilde{H}_{r}^{2}(D)}^{2} .
$$

Now by a standard Sobolev inequality for $H^{1}(E)$,

$$
|w(r, z)| \leq C\left(\left\|\partial_{z} w\right\|_{L^{2}(E)}^{2}+\|w\|_{L^{2}(E)}^{2}\right) .
$$

Applying Lemma 3.2 again to bound $\|w\|_{L^{2}(E)}^{2}$ and combining these inequalities completes the proof of the proposition.

It is easy to see that $\widetilde{\Pi}_{h}^{\theta} w$ is a continuous function on $D$ and vanishes on $\partial D$. We will now prove an approximation estimate for $\widetilde{\Pi}_{h}^{\theta}$ using the following lemma.

Lemma 3.3. For all $v \in \widetilde{H}_{r}^{2}(D)$,

$$
\sum_{K \in \mathcal{T}_{h}}\left|\widetilde{\Pi}_{h}^{\theta} v\right|_{\widetilde{H}_{r}^{2}(K)}^{2} \leq 12|v|_{\widetilde{H}_{r}^{2}(D)}^{2} .
$$

Theorem 3.2. Let $\|v\|_{a_{\theta}}=a_{\theta}(v, v)^{1 / 2}$. For all $v \in \widetilde{H}_{r}^{2}(D)$,

$$
\left\|v-\widetilde{\Pi}_{h}^{\theta} v\right\|_{a_{\theta}} \leq C h|v|_{\widetilde{H}_{r}^{2}(D)} .
$$

Consequently, if $u$ satisfies (3.3) and $u_{h}$ satisfies (3.9), then

$$
\left\|u-u_{h}\right\|_{a_{\theta}} \leq C h\|f\|_{L_{r}^{2}(D)} .
$$


Proof. Let $\varepsilon=v-\widetilde{\Pi}_{h}^{\theta} v$. Since $r^{-1} \partial_{r}(r \varepsilon)$ satisfies (2.16) and $\partial_{z}(r \varepsilon)$ satisfies (2.13), by Lemma 2.1, we have

$$
\begin{aligned}
\left\|r^{-1} \partial_{r}(r \varepsilon)\right\|_{L_{r}^{2}(K)}^{2} & \leq C h^{2}\left|r^{-1} \partial_{r}(r \varepsilon)\right|_{H_{r}^{1}(K)}^{2}, \\
\left\|\partial_{z} \varepsilon\right\|_{L_{r}^{2}(K)}^{2} & \leq C h^{2}\left|\partial_{z} \varepsilon\right|_{H_{r}^{1}(K)}^{2} .
\end{aligned}
$$

Summing over all elements, we have

$$
\left\|v-\widetilde{\Pi}_{h}^{\theta} v\right\|_{a_{\theta}}^{2} \leq C \sum_{K \in \mathcal{T}_{h}} h^{2}\left|v-\widetilde{\Pi}_{h}^{\theta} v\right|_{\widetilde{H}_{r}^{2}(K)}^{2} .
$$

Using Lemma 3.3 we finish the proof of the first inequality of the theorem. The second follows from the first by the orthogonality property of the Galerkin method and the regularity estimate of Theorem 3.1 .

\section{The azimuthal Maxwell problem: Bilinear elements}

In this section, we consider the same Maxwell problem and the same weak formulation as the one in the previous section. However, we study a different discretization. We discretize (3.3) using the standard bilinear finite element space

$$
V_{h}^{\theta}=\left\{v \in V^{\theta}:\left.v\right|_{K} \text { is bilinear in } r \text { and } z \text { for all } K \in \mathcal{T}_{h}\right\},
$$

instead of $\widetilde{V}_{h}^{\theta}$. Now, instead of (3.9), the finite element solution is defined as the unique function $u_{h} \in V_{h}^{\theta}$ satisfying

$$
a_{\theta}\left(u_{h}, v_{h}\right)=\left(f, v_{h}\right)_{r} \quad \text { for all } v_{h} \in V_{h}^{\theta} .
$$

Considering that bilinear elements are standard and may be easier to implement than the elements of the previous section, this discretization may be preferred over (3.9).

To analyze the bilinear element, we shall need a stronger regularity result. This is because it is not possible to control the error in nodal bilinear interpolation of $u$ using the norm $|u|_{\widetilde{H}_{r}^{2}(D)}$. Indeed, if $u=1 / r$ on an element $K$ away from $\Gamma_{0}$, then $|u|_{\widetilde{H}_{r}^{2}(K)}=0$, but the error in the bilinear interpolation is not zero. Such a problem did not arise in the previous sections. We will overcome this problem by controlling the interpolation error using an additional derivative of $u$, namely $\partial_{r r} u$. In order to do this, we first prove a regularity result stronger than Theorem 3.1 whereby such derivatives can be controlled by data.

4.1. A regularity estimate. To prove an improvement of Theorem 3.1, we begin by showing that solutions of (3.3) can be approximated by smooth functions. Smooth approximating functions are particularly easy to construct in the case of our simple geometry. Let $J_{\nu}(r)$ denote the Bessel function of the first kind of order $\nu$. Define

$$
j_{m}(r)=\frac{\sqrt{2}}{\left|J_{2}\left(\beta_{m}\right)\right|} J_{1}\left(\beta_{m} r\right),
$$

where $\beta_{m}$ denotes the $m$ th positive zero of $J_{1}$. Define

$$
s_{n}(z)=\sqrt{2} \sin (n \pi z) .
$$

Then by classical completeness results for Bessel functions (see [12, Ch. 8], cf. [16, $\S 18.5]$ ) the set of functions $e_{m n}(r, z)=j_{m}(r) s_{n}(z)$ for all $m, n \in \mathbb{N}$ forms a complete orthonormal basis for $L_{r}^{2}(D)$. These functions are eigenfunctions of $\mathcal{L}_{\theta}$. Indeed, 
letting $\lambda_{m n}=\beta_{m}^{2}+(n \pi)^{2}$, we have $\mathcal{L}_{\theta} e_{m n}=\lambda_{m n} e_{m n}$. Consequently, expanding $u$ and $f$ in terms of $e_{m n}$,

$$
u=\sum_{m, n \in \mathbb{N}} c_{m n} e_{m n} \text { and } f=\sum_{m, n \in \mathbb{N}} d_{m n} e_{m n},
$$

we observe that $\mathcal{L}_{\theta} u=f$ implies $c_{m n}=d_{m n} / \lambda_{m n}$. Now consider the partial sums

$$
u_{\ell}=\sum_{m, n=0}^{\ell} c_{m n} e_{m n} \quad \text { and } \quad f_{\ell}=\sum_{m, n=0}^{\ell} d_{m n} e_{m n} .
$$

The functions $u_{\ell}$ are smooth approximations to $u$ as shown below.

Proposition 4.1. The sequence $u_{\ell}$ converges to $u$ in $\widetilde{H}_{r}^{2}(D)$.

Proof. Clearly $u-u_{\ell}$ satisfies

$$
\begin{aligned}
\mathcal{L}_{\theta}\left(u-u_{\ell}\right) & =f-f_{\ell}, & & \text { on } D, \\
u-u_{\ell} & =0, & & \text { on } \partial D,
\end{aligned}
$$

By Theorem 3.1

$$
\left\|u-u_{\ell}\right\|_{\widetilde{H}_{r}^{2}(D)} \leq C\left\|f-f_{\ell}\right\|_{L_{r}^{2}(D)}
$$

Since $f_{\ell} \rightarrow f$ in $L_{r}^{2}(D)$, we have the result.

We can now give the improvement of Theorem 3.1 .

Theorem 4.1. If $u$ satisfies (3.3) for some $f \in L_{r}^{2}(D)$, then

$$
\|u\|_{H_{r}^{2}(D)}+\|u\|_{\widetilde{H}_{r}^{2}(D)}+\left\|\partial_{r}\left(\frac{u}{r}\right)\right\|_{L_{r}^{2}(D)} \leq C\|f\|_{L_{r}^{2}(D)} .
$$

Proof. Let $u_{\ell}$ be as in Proposition 4.1 and $g_{\ell}=\partial_{r} r^{-1} \partial_{r}\left(r u_{\ell}\right)$. Our proof is based on the identity

$$
\partial_{r}\left(\frac{u_{\ell}}{r}\right)=-\frac{1}{r^{3}} \int_{0}^{r} s^{2} g_{\ell}(s, z) \mathrm{d} s .
$$

This identity follows by integration by parts:

$$
\begin{aligned}
-\frac{1}{r^{3}} \int_{0}^{r} s^{2} g_{\ell}(s, z) \mathrm{d} s & =-\frac{1}{r^{3}}\left(r \partial_{r}\left(r u_{\ell}\right)-\int_{0}^{r} 2 \partial_{r}\left(r u_{\ell}\right)(s, z) \mathrm{d} s\right) \\
& =-\frac{1}{r^{3}}\left(r \partial_{r}\left(r u_{\ell}\right)-2 r u_{\ell}\right)=\partial_{r}\left(u_{\ell} / r\right) .
\end{aligned}
$$

The operations above are justified because $u_{\ell}$ is smooth. Now we apply the Hardy inequality [11,

$$
\int_{0}^{1} r^{\alpha}\left|\frac{1}{r} \int_{0}^{r} F(s) \mathrm{d} s\right|^{2} \mathrm{~d} r \leq \frac{4}{(1-\alpha)^{2}} \int_{0}^{1} r^{\alpha}|F(r)|^{2} \mathrm{~d} r
$$

with $\alpha=-3$ and $F(s)=s^{2} g_{\ell}(s, z)$ to bound the right hand side of (4.3). This yields

$$
\left\|\partial_{r}\left(\frac{u_{\ell}}{r}\right)\right\|_{L_{r}^{2}(D)}^{2} \leq \frac{1}{4}\left\|g_{\ell}\right\|_{L_{r}^{2}(D)}^{2}
$$

Since $f_{\ell}=-g_{\ell}-\partial_{z z} u_{\ell}$ and since Theorem 3.1 gives $\left\|\partial_{z z} u_{\ell}\right\|_{L_{r}^{2}(D)} \leq C\left\|f_{\ell}\right\|_{L_{r}^{2}(D)}$, we have

$$
\left\|\partial_{r}\left(\frac{u_{\ell}}{r}\right)\right\|_{L_{r}^{2}(D)}^{2} \leq C\|f\|_{L_{r}^{2}(D)}^{2}
$$


Now, by standard arguments using Proposition 4.1, it follows that the distributional derivative $\partial_{r}(u / r)$ exists in $L_{r}^{2}(D)$ and

$$
\left\|\partial_{r}\left(\frac{u}{r}\right)\right\|_{L_{r}^{2}(D)}^{2} \leq C\|f\|_{L_{r}^{2}(D)}^{2} .
$$

Now it only remains to prove that

$$
\left\|\partial_{r r} u\right\|_{L_{r}^{2}(D)} \leq C\|f\|_{L_{r}^{2}(D)} .
$$

This follows from (4.5) and the identity

$$
\partial_{r r} u=\partial_{r} r^{-1} \partial_{r}(r u)-\partial_{r}(u / r)
$$

since $\left\|\partial_{r} r^{-1} \partial_{r}(r u)\right\|_{L_{r}^{2}(D)} \leq C\|f\|_{L_{r}^{2}(D)}$ by Theorem 3.1 ,

Remark 4.1. Note that although Theorem 4.1 shows that the second derivatives $\partial_{r r} u, \partial_{r z} u$ and $\partial_{z z} u$ are in $L_{r}^{2}(D)$, it is not true in general that $u^{\Omega} \in H^{2}(\Omega)$. This is because $\breve{H}^{2}(\Omega)$ is not isomorphic to $H_{r}^{2}(D)$ (see 3 for more results in this direction). Indeed, as indicated by (2.10), one would also need $\partial_{r} u \in L_{1 / r}^{2}(D)$ for $u^{\Omega}$ to be in $\breve{H}^{2}(\Omega)$, a condition not given by Theorem 4.1

4.2. An approximation estimate. Now consider the finite element approximation defined by (4.2) using the bilinear space $V_{h}^{\theta}$ defined in (4.1). First we prove approximation properties of $V^{\theta}$ in the required norms using the nodal interpolant $\Pi_{h}^{\theta}: \widetilde{H}_{r}^{2}(D) \mapsto V_{h}^{\theta}$. On every element $K,\left.\left(\Pi_{h} v\right)\right|_{K}$ is defined as the unique function in the span of $1, r, z$, and $r z$ satisfying

$$
\left(\Pi_{h}^{\theta} v\right)\left(r_{i}, z_{j}\right)=v\left(r_{i}, z_{j}\right), \quad i, j=0,1
$$

where the notation for coordinates of vertices of $K$ are as in (2.12). Proposition 3.2 shows that $\Pi_{h}^{\theta}$ is a well defined operator on $\widetilde{H}_{r}^{2}(D)$. Error estimates for this interpolant and the resulting finite element error estimates are given by the next theorem.

Theorem 4.2. For all $v \in H_{r}^{2}(D) \cap \widetilde{H}_{r}^{2}(D)$, we have

$$
\left\|v-\Pi_{h}^{\theta} v\right\|_{a_{\theta}} \leq C h\left(|v|_{H_{r}^{2}(D)}+|v|_{\widetilde{H}_{r}^{2}(D)}\right) .
$$

Consequently, if $u$ satisfies (3.3) and $u_{h}$ satisfies (4.2), then

$$
\left\|u-u_{h}\right\|_{a_{\theta}} \leq C h\|f\|_{L_{r}^{2}(D)} .
$$

Proof. Let $\varepsilon=u-\Pi_{h}^{\theta} u$. First let us estimate $\varepsilon$ on elements $K$ with an edge on the $\{r=0\}$ axis. A simple calculation shows that

$$
\left.\left(\widetilde{\Pi}_{h}^{\theta} v\right)\right|_{K}=\left.\left(\Pi_{h}^{\theta} v\right)\right|_{K}
$$

on all such elements (where $\widetilde{\Pi}_{h}^{\theta}$ is as defined in (3.10) $)$. Consequently, by the same arguments as in the proof of Theorem 3.2 , we have

$$
|\varepsilon|_{\widetilde{H}_{r}^{1}(K)} \leq C h|v|_{\widetilde{H}_{r}^{2}(K)} .
$$

Now consider the remaining elements. By Proposition 3.1.

$$
C\|\varepsilon\|_{a_{\theta}} \leq|\varepsilon|_{H_{r}^{1}(D)}+\|\varepsilon\|_{L_{1 / r}^{2}(D)} .
$$

On the elements under consideration,

$$
\left.\left(\Pi_{h} v\right)\right|_{K}=\left.\left(\Pi_{h}^{\theta} v\right)\right|_{K},
$$


where $\Pi_{h}$ is as defined in Section 2. Consequently, by the same arguments as in Theorem 2.2, we obtain that

$$
|\varepsilon|_{H_{r}^{1}(D)} \leq C h|\varepsilon|_{H_{r}^{2}(D)}
$$

Therefore, it only remains to estimate $\|\varepsilon\|_{L_{1 / r}^{2}(D)}$. If $K$ has coordinates as in (2.12), by standard estimates (in nonweighted Sobolev spaces) for the interpolant, we have

$$
\|\varepsilon\|_{L_{1 / r}^{2}(K)}^{2} \leq C \frac{1}{r_{0}}\|\varepsilon\|_{L^{2}(K)}^{2} \leq C \frac{1}{r_{0}} h^{4}|u|_{H^{2}(K)}^{2} \leq C h^{2}\left(\frac{h^{2}}{r_{0}^{2}}\right)|u|_{H_{r}^{2}(K)}^{2} .
$$

Since $h / r_{0} \leq 1$ for the elements under consideration now, the interpolation error estimate of the theorem follows. The finite element error estimate follows as a corollary using also Theorem 4.1 .

\section{MUltigRid ANALYSis}

All the discretizations that we considered in the previous sections were based on the mesh $\mathcal{T}_{h}$. Now we assume the standard geometric multigrid setting. The mesh on which a solution is sought, namely $\mathcal{T}_{h} \equiv \mathcal{T}_{J}$, is obtained by successive refinements of a coarse mesh $\mathcal{T}_{1}$. We assume that $\mathcal{T}_{1}$ is a mesh of congruent square elements, and $\mathcal{T}_{k}$ is obtained from $\mathcal{T}_{k-1}(k \geq 2)$ by dividing each square element of $\mathcal{T}_{k-1}$ into four congruent squares. We will analyze the convergence of the multigrid $\mathrm{V}$-cycle algorithm in this setting for all of the previous applications.

Let $V_{k}$ denote one of the previously defined finite element spaces $V_{h}, V_{h}^{\theta}$, or $\widetilde{V}_{h}^{\theta}$ on the mesh $\mathcal{T}_{k}$. Then $V_{k}$ is a subspace of the Sobolev space $V$ which we take to be as defined by (2.3) for the Laplace application, and as defined by (3.2) for the Maxwell application. Then

$$
V_{1} \subset V_{2} \subset \cdots \subset V_{J} \subset V
$$

Let $a(\cdot, \cdot)$ denote one of the previously defined bilinear forms on $V \times V\left(a\right.$ is either $a_{r}$ or $a_{\theta}$ ). To state the multigrid V-cycle in a form suitable for both the applications, define $Q_{k}: L_{r}^{2}(D) \mapsto V_{k}, P_{k}: V \mapsto V_{k}$, and $A_{k}: V_{k} \mapsto V_{k}$ by

$$
\begin{array}{ll}
\left(Q_{k} w, \phi\right)_{r}=(w, \phi)_{r}, & \text { for all } \phi \in V_{k} \text { and } w \in L_{r}^{2}(D), \\
a\left(P_{k} w, \phi\right)=a(w, \phi), & \text { for all } \phi \in V_{k} \text { and } w \in V, \\
\left(A_{k} w, \phi\right)_{r}=a(w, \phi), & \text { for all } \phi \text { and } w \in V_{k} .
\end{array}
$$

We want to compute the finite element solution $u_{h}=A_{J}^{-1} Q_{J} f$ efficiently using the standard V-cycle multigrid algorithm. For any $g \in V_{J}$, the V-cycle iterates $v^{(i)}$ approximating $v=A_{J}^{-1} g$ satisfy the linear iteration

$$
v^{(i+1)}=v^{(i)}+B_{J}\left(g-A_{J} g\right),
$$

where $B_{J}: V_{J} \mapsto V_{J}$ is the operator defined recursively below.

(1) $B_{1}=A_{1}^{-1}$.

(2) If $k>1$, define $B_{k}: V_{k} \mapsto V_{k}$ by $B_{k} g=v^{1}$, where $v^{1}$ is computed as follows.

(a) Pre-smoothing: $v^{1 / 3}=R_{k}^{t} g$.

(b) Coarse grid correction:

$$
v^{2 / 3}=v^{1 / 3}+B_{k-1} Q_{k-1}\left(g-A_{k} v^{1 / 3}\right) .
$$

(c) Post-smoothing: $v^{1}=v^{2 / 3}+R_{k}\left(g-A_{k} v^{2 / 3}\right)$. 
In the above algorithm, the operator $R_{k}$ represents a point Gauss-Seidel or Jacobi smoother and $R_{k}^{t}$ denotes its adjoint with respect to $(\cdot, \cdot)_{r}$. Specifically, $R_{k}$ is defined using a splitting of the finite element space as follows. Let $N_{k}=\operatorname{dim}\left(V_{k}\right)$ and let $x_{i}, i=1,2, \ldots, N_{k}$, be any enumeration of the vertices of the mesh $\mathcal{T}_{k}$ which are degrees of freedom. Let $D_{k, i}$ denote the domain formed by the four or two elements connected to the vertex $x_{i}$. Let $V_{k, i}$ denote the set of functions in $V_{k}$ which are supported on $D_{k, i}$. Define $Q_{k, i}: L_{r}^{2}\left(D_{k, i}\right) \mapsto V_{k, i}, P_{k, i}: V \mapsto V_{k, i}$, and $A_{k, i}: V_{k, i} \mapsto V_{k, i}$, by

$$
\begin{array}{ll}
\left(Q_{k, i} w, \phi\right)_{r}=(w, \phi)_{r}, & \text { for all } \phi \in V_{k, i} \text { and } w \in L_{r}^{2}\left(D_{k, i}\right), \\
a\left(P_{k, i} w, \phi\right)=a(w, \phi), & \text { for all } \phi \in V_{k, i} \text { and } w \in V, \\
\left(A_{k, i} w, \phi\right)_{r}=a(w, \phi), & \text { for all } \phi \text { and } w \in V_{k, i} .
\end{array}
$$

Define the the additive Jacobi smoother by

$$
\partial_{k}=\sum_{j=1}^{N_{k}} A_{k, j}^{-1} Q_{k, j},
$$

and the multiplicative Gauss-Seidel smoother by

$$
\mathcal{G}_{k}=\left(I-\left(I-P_{k, N_{k}}\right)\left(I-P_{k, N_{k}-1}\right) \cdots\left(I-P_{k, 1}\right)\right) A_{k}^{-1} .
$$

We can set $R_{k}$ in the V-cycle to either $\alpha \mathcal{J}_{k}$ for some scaling factor $\alpha$, or $\mathcal{G}_{k}$.

Remark 5.1. In the above presentation of the multigrid algorithm, it appears that one is required to compute the action of $Q_{k-1}$. The mass matrix inversion associated with this can be avoided in implementation due to the special form of the smoothers. In addition, the action of $\mathcal{G}_{k}$ is not implemented as presented in (5.2). In fact, its implementation avoids the computation of $A_{k}^{-1}$. For details on these implementation issues, see, e.g., [7.

Local estimates enable us to prove the following basic two sided bound on the additive operator. This will be an important ingredient in the multigrid analysis. Similar estimates are well known for standard applications in nonweighted Sobolev spaces.

Lemma 5.1. There is a constant $C_{\mathfrak{J}}$ independent of $k$ such that for all $v \in V_{k}$,

$$
\frac{1}{4} a(v, v) \leq\left(\mathcal{J}_{k}^{-1} v, v\right)_{r} \leq C_{\mathfrak{\jmath}} \frac{1}{h_{k}^{2}}(v, v)_{r},
$$

where $h_{k}$ is the mesh size of $\mathcal{T}_{k}$.

With the help of this lemma, we can now prove the uniform convergence of the $\mathrm{V}$-cycle for the axisymmetric Laplace and Maxwell equations.

Theorem 5.1. The multigrid $V$-cycle with the smoother $R_{k}$ set to either the scaled Jacobi smoother $\alpha \mathcal{J}_{k}$ with $0<\alpha<1 / 2$ or the Gauss-Seidel smoother $\mathcal{G}_{k}$ converges at a rate independent of $h$ : There is a $\delta<1$ independent of $k$ such that

$$
0 \leq a\left(\left(I-B_{k} A_{k}\right) v, v\right) \leq \delta a(v, v), \quad \text { for all } v \in V_{k} .
$$

Proof. The case of the Jacobi smoother. According to [8, Lemma 2.1], once we prove that the spectrum

$$
\sigma\left(I-R_{k} A_{k}\right) \subseteq[-\theta, 1), \quad \text { for all } k,
$$


and

$$
\left(R_{k}^{-1}\left(I-P_{k-1}\right) v,\left(I-P_{k-1}\right) v\right)_{r} \leq C_{P}\left\|v-P_{k-1}\right\|_{a}^{2}, \quad \text { for all } v \in V_{k},
$$

then (5.3) follows with

$$
\delta=\frac{\gamma C_{P}}{1+\gamma C_{P}}, \quad \text { with } \gamma=\max \left(\frac{1}{2}, \frac{\theta^{2}}{1-\theta}\right) .
$$

To verify (5.4), note that by Lemma [5.1, $\sigma\left(\alpha \mathcal{J}_{k} A_{k}\right) \subset(0,4 \alpha]$, so

$$
\sigma\left(I-\alpha \mathcal{J}_{k} A_{k}\right) \subset[1-4 \alpha, 1) .
$$

If we choose $0<\alpha<1 / 2$, then (5.4) holds with $\theta=4 \alpha-1$.

To verify the second condition (5.5), we first use a duality argument. Let $w \in V$ satisfy

$$
a(w, \phi)=\left(v-P_{k-1} v, \phi\right)_{r}, \quad \text { for all } \phi \in V .
$$

Then, using Theorems 2.2 3.2, or 4.2, as appropriate,

$$
\left\|w-P_{k-1} w\right\|_{a} \leq C h_{k}\left\|v-P_{k-1} v\right\|_{L_{r}^{2}(D)} .
$$

Hence,

$$
\begin{aligned}
\left(v-P_{k-1} v, v-P_{k-1} v\right)_{r} & =a\left(w, v-P_{k-1} v\right) \\
& =a\left(w-P_{k-1} w, v-P_{k-1} v\right) \\
& \leq\left\|w-P_{k-1} w\right\|_{a}\left\|v-P_{k-1} v\right\|_{a} \\
& \leq C h\left\|v-P_{k-1} v\right\|_{L_{r}^{2}(D)}\left\|v-P_{k-1} v\right\|_{a} .
\end{aligned}
$$

Thus,

$$
\left\|v-P_{k-1} v\right\|_{L_{r}^{2}(D)} \leq C h_{k}\left\|v-P_{k-1} v\right\|_{a} .
$$

The proof of (5.5) can now be completed using (5.7). Indeed,

$$
\begin{aligned}
\left(\alpha^{-1} \mathcal{J}_{k}^{-1}\left(I-P_{k-1}\right) v,\left(I-P_{k-1}\right) v\right)_{r} & \leq \frac{C_{\mathfrak{J}}}{\alpha h_{k}^{2}}\left\|v-P_{k-1} v\right\|_{L_{r}^{2}(D)}^{2}, & & \text { by Lemma 5.1, } \\
& \leq C\left\|v-P_{k-1} v\right\|_{a}^{2}, & & \text { by (5.7), }
\end{aligned}
$$

so (5.5) holds.

The case of the Gauss-Seidel smoother. According to [8, Lemma 2.2], once we verify that

$$
\left\|I-R_{k} A_{k}\right\|_{a} \leq 1
$$

and

$$
\left(\bar{R}_{k}^{-1}\left(I-P_{k-1}\right) v, v-P_{k-1} v\right)_{r} \leq C_{M}\left\|\left(I-P_{k-1}\right)\right\|_{a}^{2}, \quad \text { for all } v \in V_{k},
$$

where $\bar{R}_{k}=R_{k}+R_{k}^{t}-R_{k}^{t} A_{k} R_{k}$, the convergence estimate (5.3) follows with

$$
\delta=\frac{C_{M}}{1+C_{M}} .
$$

Inequality (5.8) follows from the product representation

$$
I-\mathcal{G}_{k} A_{k}=\left(I-P_{k, N_{k}}\right) \cdots\left(I-P_{k, 1}\right),
$$


so it suffices to prove (5.9). Using standard arguments 7 and the fact that a basis function "interacts" with at most eight other basis functions, it is easy to see that

$$
\sum_{i=1}^{N_{k}} a\left(P_{k, i} v, v\right) \leq 9^{2} a\left(\overline{\mathcal{G}}_{k} A_{k} v, v\right) .
$$

Since (5.11) implies that $a\left(\mathfrak{J}_{k} A_{k} v, v\right) \leq 9^{2} a\left(\overline{\mathfrak{G}}_{k} A_{k} v, v\right)$, by Lemma 5.1 we obtain

$$
\frac{h_{k}^{2}}{C_{\mathfrak{J}}}(w, w)_{r} \leq\left(\mathcal{J}_{k} w, w\right)_{r} \leq 9^{2}\left(\overline{\mathfrak{G}}_{k} w, w\right)_{r}, \quad \text { for all } w \in V_{k} .
$$

Consequently,

$$
\begin{array}{rlr}
\left(\overline{\mathcal{G}}_{k}^{-1}\left(v-P_{k-1} v\right), v-P_{k-1} v\right)_{r} & \leq \frac{C_{\mathfrak{J}} 9^{2}}{h_{k}^{2}}\left\|v-P_{k-1}\right\|_{L_{r}^{2}(D)}^{2} \\
& \leq C\left\|v-P_{k-1} v\right\|_{a}^{2}, \quad \text { by (5.7), }
\end{array}
$$

so (5.9) follows.

\section{NumericAl RESUlts}

In this section, we give the results of numerical experiments illustrating the convergence rates of the $\mathrm{V}$-cycle multigrid algorithm. We report three numerical experiments, one for the axisymmetric Laplace problem and two for the azimuthal Maxwell problem. In all cases, we divide the unit square into $n \times n$ square elements with $n=2^{k}$ and use the finite element discretizations which we have previously discussed. The boundary conditions are as described in the previous sections.

We implemented the V-cycle multigrid algorithm with one sweep of point GaussSeidel smoothing as described in Section 5 for all cases. One can use the V-cycle multigrid operator $B_{J}$ in two ways. One obvious way to use it is in the linear iteration (5.1). By Theorem [5.1, this iteration will converge at a rate independent of the number of unknowns. The rate of convergence in the energy norm is at most $\delta_{J} \equiv\left\|I-B_{J} A_{J}\right\|_{a}$. Another way to use the V-cycle is to use $B_{J}$ as a preconditioner for $A_{J}$ in a preconditioned conjugate gradient iteration. In this case the convergence is determined by the condition number of the preconditioned system $\kappa\left(B_{J} A_{J}\right)$. Note that by Theorem 5.1 ,

$$
\left(1-\delta_{J}\right) a(v, v) \leq a(B A v, v) \leq a(v, v),
$$

so $\kappa\left(B_{J} A_{J}\right) \leq 1 /\left(1-\delta_{J}\right)$. Since $\delta_{J}$ is bounded away from one independently of $J$, the condition number is bounded from above independently of $J$.

TABLE 6.1. V-cycle convergence rates

\begin{tabular}{|c|c|c|c|c|c|c|}
\hline \multirow{4}{*}{$J$} & \multicolumn{3}{|c|}{ Bilinear elements } & \multicolumn{2}{c|}{$\langle r, 1 / r, z, z / r\rangle$-elements } \\
\cline { 2 - 7 } & \multicolumn{2}{|c|}{ Laplace equation } & \multicolumn{2}{c|}{ Maxwell equation } & \multicolumn{2}{c|}{ Maxwell equation } \\
\cline { 2 - 7 } & $\kappa\left(B_{J} A_{J}\right)$ & $\left\|I-B_{J} A_{J}\right\|_{a_{r}}$ & $\kappa\left(B_{J} A_{J}\right)$ & $\left\|I-B_{J} A_{J}\right\|_{a_{\theta}}$ & $\kappa\left(B_{J} A_{J}\right)$ & $\left\|I-B_{J} A_{J}\right\|_{a_{\theta}}$ \\
\hline 2 & 1.13 & 0.12 & 1.08 & 0.08 & 1.08 & 0.08 \\
3 & 1.19 & 0.16 & 1.17 & 0.14 & 1.17 & 0.14 \\
4 & 1.20 & 0.17 & 1.20 & 0.17 & 1.20 & 0.17 \\
5 & 1.21 & 0.17 & 1.21 & 0.17 & 1.21 & 0.17 \\
6 & 1.21 & 0.17 & 1.21 & 0.17 & 1.21 & 0.17 \\
7 & 1.21 & 0.17 & 1.21 & 0.17 & 1.21 & 0.17 \\
8 & 1.21 & 0.17 & 1.21 & 0.17 & 1.21 & 0.17 \\
9 & 1.21 & 0.17 & 1.21 & 0.17 & 1.21 & 0.17 \\
10 & 1.21 & 0.17 & 1.21 & 0.17 & 1.21 & 0.17 \\
\hline
\end{tabular}


In Table 6.1. we report the values of $\kappa\left(B_{J} A_{J}\right)$ and the convergence rate $\left\|I-B_{J} A_{J}\right\|_{a}$. These values were computed using estimates of the largest and smallest eigenvalues of $B_{J} A_{J}$ provided by a few iterations of the Lanczos method. As we see from the tables, the observed convergence rates are almost identical and clearly demonstrate a rate of convergence independent of the number of levels.

\section{Appendix A. Proofs of the lemmas}

Proof of Lemma 2.1, In view of Proposition 2.1, it suffices to prove the required estimate for smooth functions $v$.

(i) Suppose (2.13) holds. Integrating the equation

$$
v(r, z)-v\left(r_{1}, Z\right)=-\int_{r}^{r_{1}} \partial_{r} v(\rho, Z) \mathrm{d} \rho+\int_{Z}^{z} \partial_{z} v(r, \zeta) \mathrm{d} \zeta
$$

over $Z \in\left(z_{0}, z_{1}\right)$ and using (2.13), we obtain

$$
\begin{aligned}
h v(r, z) & =-\int_{z_{0}}^{z_{1}} \int_{r}^{r_{1}} \partial_{r} v(\rho, Z) \mathrm{d} \rho \mathrm{d} Z+\int_{z_{0}}^{z_{1}} \int_{Z}^{z} \partial_{z} v(r, \zeta) \mathrm{d} \zeta \mathrm{d} Z, \quad \text { so } \\
h^{2}|v(r, z)|^{2} & \leq\left.\left. 2 h \ln \left(\frac{r_{1}}{r}\right) \int_{z_{0}}^{z_{1}}\left|\int_{r}^{r_{1}} \rho\right| \partial_{r} v(\rho, Z)\right|^{2} \mathrm{~d} \rho\left|\mathrm{d} Z+h^{2} \int_{z_{0}}^{z_{1}} \int_{Z}^{z}\right| \partial_{z} v(r, \zeta)\right|^{2} \mathrm{~d} \zeta \mathrm{d} Z .
\end{aligned}
$$

Hence,

$$
h^{2}\|v\|_{L_{r}^{2}(K)}^{2} \leq 2 h^{2}\left\|\partial_{r} v\right\|_{L_{r}^{2}(K)}^{2}\left(\int_{r_{0}}^{r_{1}} r \ln \frac{r_{1}}{r} \mathrm{~d} r\right)+h^{4}\left\|\partial_{z} v\right\|_{L_{r}^{2}(K)}^{2} .
$$

It is easy to show that

$$
\int_{r_{0}}^{r_{1}} r \ln \frac{r_{1}}{r} \mathrm{~d} r \begin{cases}=h^{2} / 4 & \text { if } r_{0}=0 \\ \leq h^{2} / 2 & \text { if } r_{0}>0\end{cases}
$$

so the result follows.

(ii) Suppose (2.14) holds. Then, since

$$
v(r, z)=v\left(R, z_{0}\right)+\int_{z_{0}}^{z} \partial_{z} v(R, \zeta) \mathrm{d} \zeta+\int_{R}^{r} \partial_{r} v(\rho, z) d \rho,
$$

integrating over $R \in\left(r_{0}, r_{1}\right)$ and utilizing (2.14), we find that

$$
\begin{aligned}
h v(r, z)= & \int_{r_{0}}^{r_{1}} \int_{z_{0}}^{z} \partial_{z} v(R, \zeta) d R \mathrm{~d} \zeta+\int_{r_{0}}^{r_{1}} \int_{R}^{r} \partial_{r} v(\rho, z) \partial \rho \mathrm{d} R, \quad \text { so } \\
h^{2}|v(r, z)|^{2} \leq & 2 h \ln \left(\frac{r_{1}}{r_{0}}\right)\left\|\partial_{z} v\right\|_{L_{r}^{2}(K)}^{2} \\
& +2\left(\int_{r_{0}}^{r_{1}}\left|\ln \frac{r}{R}\right| \mathrm{d} R\right)\left(\left.\int_{r_{0}}^{r_{1}}\left|\int_{R}^{r} \rho \partial_{r}\right| v(\rho, z)\right|^{2} \partial \rho \mid \mathrm{d} R\right) .
\end{aligned}
$$

Since $\ln \left(r_{1} / r_{0}\right)=\ln \left(1+\left(h / r_{0}\right)\right) \leq h / r_{0}$ and

$$
\int_{r_{0}}^{r_{1}} \int_{r_{0}}^{r_{1}} r\left|\ln \frac{r}{R}\right| \mathrm{d} R \mathrm{~d} r=r_{0} r_{1} h+\frac{5}{12} h^{3}-\ln \left(\frac{r_{1}}{r_{0}}\right)\left(r_{0}^{3}+\frac{1}{2}\left(3 r_{0}^{2} h+r_{0} h^{2}\right)\right) \leq \frac{11 h^{3}}{12}
$$

we obtain after further integration that

$$
h^{2}\|v\|_{L_{r}^{2}(K)}^{2}<\frac{3}{2} h^{4}\left\|\partial_{z} v\right\|_{L_{r}^{2}(K)}^{2}+2 h^{4}\left\|\partial_{r} v\right\|_{L_{r}^{2}(K)}^{2},
$$

from which the needed estimate follows. 
(iii) Suppose (2.15) holds. Multiplying (A.1) by $R^{1 / 2}$ and integrating over $R$ gives

$$
\frac{2}{3} h^{3 / 2} v(r, z)=\int_{0}^{r_{1}} \int_{z_{0}}^{z} R^{1 / 2} \partial_{z} v(R, \zeta) \mathrm{d} \zeta \mathrm{d} R+\int_{0}^{r_{1}} \int_{R}^{r} R^{1 / 2} \partial_{r} v(\rho, z) \mathrm{d} \rho \mathrm{d} R .
$$

Proceeding as before, we find that

$$
\frac{4}{9} h^{3}\|v\|_{L_{r}^{2}(K)}^{2} \leq h^{5}\left\|\partial_{z} v\right\|_{L_{r}^{2}(K)}^{2}+h^{2}\left\|\partial_{r} v\right\|_{L_{r}^{2}(K)}^{2} \int_{0}^{r_{1}} \int_{0}^{r_{1}} r\left|\ln \frac{r}{R}\right| \mathrm{d} R \mathrm{~d} r .
$$

The integral can be evaluated by taking the limit as $r_{0} \rightarrow 0$ in the equality of (A.2). Then we get the required inequality.

(iv) Proof of the last case is similar to the one above.

Proof of Lemma 2.2. It is easy to verify that

$$
\partial_{r z}\left(\Pi_{h} v\right)=\left\{\begin{aligned}
\frac{3}{2 h^{5 / 2}} \int_{0}^{h} \int_{z_{0}}^{z_{1}} \rho^{1 / 2} \partial_{r z} v(\rho, \zeta) \mathrm{d} \rho \mathrm{d} \zeta & \text { if } r_{0}=0, \\
\frac{1}{h^{2}} \int_{r_{0}}^{r_{1}} \int_{z_{0}}^{z_{1}} \partial_{r z} v(\rho, \zeta) \mathrm{d} \rho \mathrm{d} \zeta & \text { if } r_{0}>0 .
\end{aligned}\right.
$$

The estimate of the lemma follows taking norms on both sides and applying the Cauchy-Schwarz inequality.

Proof of Lemma 3.1, Let $u \in \widetilde{H}_{r}^{1}(D)$. First, we extend $u$ to a function $\widetilde{u}$ on the right half plane $\mathbb{R}_{+}^{2} \equiv\{(r, z): r>0\}$ such that

$$
\|\widetilde{u}\|_{\widetilde{H}_{r}^{1}\left(\mathbb{R}_{+}^{2}\right)} \leq C\|u\|_{\widetilde{H}_{r}^{1}(D)} .
$$

This can be done as follows:

(i) Reflect $u$ about the $\{z=0\}$ line to get a function in the rectangle $\{0<$ $r<1,-1<z<1\}$. It can be easily seen that the standard arguments that prove that reflection gives bounded extensions apply to our weighted Sobolev space as well.

(ii) Next, reflect the combined function about the $\{z=1\}$ line to get a function in $\{0<r<1,-1<z<3\}$.

(iii) Now reflect about the $\{r=1\}$ line to get a function in $\{0<r<3 / 2,-1<$ $z<3\}$. Note that this reflection does not reflect the values of $u$ near the $\{r=0\}$ line to the $r>1$ side.

(iv) Finally, $\widetilde{u}$ is obtained by multiplying the function resulting from the previous steps with a smooth cut off function that is identically one in a neighborhood of $D$ and has compact support in the extended rectangle $\{0<r<3 / 2,-1<z<3\}$. This function satisfies A.3.

Next we cut off the function $\widetilde{u}$ to zero smoothly near the $\{r=0\}$ line. Let $\chi_{\varepsilon}(r) \in C^{\infty}\left(\mathbb{R}_{+}^{2}\right)$ be a cutoff function satisfying

$$
\begin{aligned}
& 0 \leq \chi_{\varepsilon} \leq 1 \\
& \chi_{\varepsilon}(r)= \begin{cases}1 & \text { if } r \geq \varepsilon, \\
0 & \text { if } r \leq \varepsilon / 2,\end{cases} \\
& \left|\partial_{r} \chi_{\varepsilon}(r)\right| \leq C / \varepsilon \quad \text { for all } r,
\end{aligned}
$$

and define

$$
\widetilde{u}_{\varepsilon}=\chi_{\varepsilon}(r) \widetilde{u}(r, z)
$$


Then, if $S_{\varepsilon}$ denotes the strip $\{(r, z): 0<r<\varepsilon\}$, we have

$$
\begin{aligned}
\left\|\widetilde{u}_{\varepsilon}-\widetilde{u}\right\|_{L_{1 / r}^{2}\left(\mathbb{R}_{+}^{2}\right)}^{2} & \leq\|\widetilde{u}\|_{L_{1 / r}^{2}\left(S_{\varepsilon}\right)}^{2}, \\
\left\|\partial_{r}\left(\widetilde{u}_{\varepsilon}-\widetilde{u}\right)\right\|_{L_{r}^{2}\left(\mathbb{R}_{+}^{2}\right)}^{2} & =\int_{\mathbb{R}_{+}^{2}}\left|\partial_{r} \widetilde{u}\left(1-\chi_{\varepsilon}\right)+\widetilde{u} \partial_{r}\left(1-\chi_{\varepsilon}\right)\right|^{2} r \mathrm{~d} r \mathrm{~d} z \\
& \leq 2\left\|\partial_{r} \widetilde{u}\right\|_{L_{r}^{2}\left(S_{\varepsilon}\right)}^{2}+C\|\widetilde{u} / r\|_{L_{r}^{2}\left(S_{\varepsilon}\right)}^{2}, \\
\left\|\partial_{z}\left(\widetilde{u}_{\varepsilon}-\widetilde{u}\right)\right\|_{L_{r}^{2}\left(\mathbb{R}_{+}^{2}\right)}^{2} & \leq\left\|\partial_{z}\left(\widetilde{u}_{\varepsilon}-\widetilde{u}\right)\right\|_{L_{r}^{2}\left(S_{\varepsilon}\right)}^{2} .
\end{aligned}
$$

Since $\|\widetilde{u}\|_{\widetilde{H}_{r}^{1}\left(S_{\varepsilon}\right)} \rightarrow 0$ as $\varepsilon \rightarrow 0$, we find that given any $\epsilon>0$ we can find $\varepsilon>0$ for which

$$
\left\|\widetilde{u}_{\varepsilon}-\widetilde{u}\right\|_{\widetilde{H}_{r}^{1}\left(\mathbb{R}_{+}^{2}\right)} \leq \epsilon
$$

Finally, we mollify the function $\widetilde{u}_{\varepsilon}$ obtained above. Let

$$
\phi_{\delta}(r, z)= \begin{cases}c_{\phi, \delta} e^{-\left(\frac{\delta^{2}}{\delta^{2}-r^{2}-z^{2}}\right)} & \text { if } r^{2}+z^{2}<\delta^{2} \\ 0 & \text { otherwise }\end{cases}
$$

where the constant $c_{\phi, \delta}$ is chosen such that $\int_{\mathbb{R}^{2}} \phi_{\delta}(r, z) \mathrm{d} r \mathrm{~d} z=1$. Define

$$
\widetilde{u}_{\varepsilon \delta}=\widetilde{u}_{\varepsilon} * \phi_{\delta} .
$$

If we choose $\delta<\varepsilon / 4$, then

$$
\left\|\widetilde{u}_{\varepsilon \delta}-\widetilde{u}_{\varepsilon}\right\|_{L_{1 / r}^{2}\left(\mathbb{R}_{+}^{2}\right)} \leq \frac{4}{\varepsilon}\left\|\widetilde{u}_{\varepsilon \delta}-\widetilde{u}_{\varepsilon}\right\|_{L^{2}\left(\mathbb{R}_{+}\right)} .
$$

Moreover, by the well known properties of mollifiers, $\left\|\widetilde{u}_{\varepsilon \delta}-\widetilde{u}_{\varepsilon}\right\|_{H^{1}\left(\mathbb{R}^{2}\right)} \rightarrow 0$ as $\delta \rightarrow 0$. Now choosing $\delta>0$ further smaller if necessary, we can ensure that

$$
\left\|\widetilde{u}_{\varepsilon \delta}-\widetilde{u}_{\varepsilon}\right\|_{L^{2}\left(\mathbb{R}_{+}\right)} \leq \varepsilon \epsilon \text { and }\left\|\widetilde{u}_{\varepsilon \delta}-\widetilde{u}_{\varepsilon}\right\|_{H^{1}\left(\mathbb{R}_{+}\right)} \leq \epsilon,
$$

so that

$$
\left\|\widetilde{u}_{\varepsilon \delta}-\widetilde{u}_{\varepsilon}\right\|_{\widetilde{H}_{r}^{1}\left(\mathbb{R}_{+}^{2}\right)} \leq 5 \epsilon .
$$

Combining this with (A.7) we conclude that for the arbitrarily given $\epsilon$, there exist $\varepsilon$ and $\delta$ such that

and $\widetilde{u}_{\varepsilon \delta} \in C^{\infty}(\bar{D}) \cap L_{1 / r}^{2}(D)$.

$$
\left\|\widetilde{u}_{\varepsilon \delta}-u\right\|_{\widetilde{H}_{r}^{1}(D)} \leq 6 \epsilon
$$

Proof of Lemma 3.2. Because of Lemma 3.1, it suffices to prove the stated inequality for $v \in C^{\infty}(\bar{D}) \cap L_{1 / r}^{2}(D)$. For such $v$,

$$
v(r, z)^{2}-v(a, z)^{2}=\int_{a}^{r} \partial_{r} v^{2}(\rho, z) \mathrm{d} \rho .
$$

Integrating with respect to $z$ we get

$$
\int_{z_{0}}^{z_{1}} v(a, z)^{2} \mathrm{~d} z=\int_{z_{0}}^{z_{1}} v(r, z)^{2} \mathrm{~d} z-\int_{z_{0}}^{z_{1}} \int_{a}^{r} 2 v(\rho, z) \partial_{r} v(\rho, z) \mathrm{d} \rho \mathrm{d} z .
$$

Applying the Cauchy-Schwarz inequality and integrating both sides with respect to $r$,

$$
\begin{aligned}
h\|v\|_{L^{2}\left(S_{a}\right)} & \leq\|v\|_{L^{2}(K)}^{2}+2 h\|v\|_{L_{1 / r}^{2}(K)}\left\|\partial_{r} v\right\|_{L_{r}^{2}(K)} \\
& \leq r_{1}\|v\|_{L_{1 / r}^{2}(K)}^{2}+h\|v\|_{L_{1 / r}^{2}(K)}^{2}+h\left\|\partial_{r} v\right\|_{L_{r}^{2}(K)}^{2} .
\end{aligned}
$$

Hence, the lemma is proved. 
Proof of Lemma 3.3. On any element $K$ (with coordinates as in (2.12)), the following identities hold:

$$
\begin{aligned}
\partial_{r} \frac{1}{r} \partial_{r}\left(r \widetilde{\Pi}_{h}^{\theta} u\right) & =0 \\
\partial_{z} \frac{1}{r} \partial_{r}\left(r \widetilde{\Pi}_{h}^{\theta} u\right) & =\frac{2}{h^{2}\left(r_{1}+r_{0}\right)} \int_{K} \partial_{r z}(r u) \mathrm{d} r \mathrm{~d} z \\
\partial_{z z} \widetilde{\Pi}_{h}^{\theta} u & =0 \\
\partial_{r z} \widetilde{\Pi}_{h}^{\theta} u & =\frac{1}{h^{2}\left(r_{1}+r_{0}\right)}\left[\left(1+\frac{r_{1} r_{0}}{r^{2}}\right) \int_{K} \partial_{r z}(r u) \mathrm{d} r \mathrm{~d} z\right. \\
& \left.-h \frac{r_{1} r_{0}}{r^{2}} \int_{z_{0}}^{z_{1}}\left(\partial_{z} u\left(r_{1}, \zeta\right)+\partial_{z} u\left(r_{0}, \zeta\right)\right) \mathrm{d} \zeta\right]
\end{aligned}
$$

These identities hold even when $r_{0}=0$. Taking $L_{r}^{2}(K)$-norms on both sides of (A.9) and applying the Cauchy-Schwarz inequality gives

$$
\left\|\partial_{z} \frac{1}{r} \partial_{r}\left(r \widetilde{\Pi}_{h}^{\theta} u\right)\right\|_{L_{r}^{2}(K)}^{2} \leq\left\|\frac{1}{r} \partial_{r z}(r u)\right\|_{L_{r}^{2}(K)}^{2} \leq|u|_{\widetilde{H}_{r}^{2}(K)}^{2} .
$$

For (A.11), we take $L_{r}^{2}(K)$-norms on both sides, apply the triangle inequality, and estimate the line integrals using Lemma 3.2 .

$\left\|\partial_{r z} \widetilde{\Pi}_{h}^{\theta} u\right\|_{L_{r}^{2}(K)}^{2} \leq 4\left\|\frac{1}{r} \partial_{r z}(r u)\right\|_{L_{r}^{2}(K)}^{2}+4 \frac{r_{1}+h}{r_{1}+r_{0}}\left\|\frac{1}{r} \partial_{z} u\right\|_{L_{r}^{2}(K)}^{2}+4 \frac{h}{r_{1}+r_{0}}\left\|\partial_{r z} u\right\|_{L_{r}^{2}(K)}^{2}$.

Summing over all elements and applying Proposition 3.1 completes the proof of the lemma.

Proof of Lemma 5.1. Since

$$
a\left(\mathcal{J}_{k} A_{k} v, v\right)=\sum_{j=1}^{N_{k}} a\left(P_{k, i} v, v\right)=\sum_{j=1}^{N_{k}} a\left(P_{k, i} v, P_{k, i} v\right) \leq \sum_{j=1}^{N_{k}}|v|_{H_{r}^{1}\left(D_{k, i}\right)}^{2} \leq 4 a(v, v),
$$

the lower inequality is proved.

To prove the upper inequality, we apply a well known characterization of additive operators [7. Specifically, for any $v \in V_{k}$ decomposed as $v=\sum_{i=1}^{N_{k}} v_{i}$ with $v_{i} \in V_{k, i}$, we have

$$
\left(\mathfrak{J}_{k}^{-1} v, v\right)=\sum_{i=1}^{N_{k}} a\left(v_{i}, v_{i}\right) .
$$

Hence, it suffices to show that

$$
\sum_{i=1}^{N_{k}} a\left(v_{i}, v_{i}\right) \leq \frac{C}{h_{k}^{2}}\|v\|_{L_{r}^{2}(D)}^{2} .
$$

This inequality will follow (by summing) once we prove the local inequality

$$
a^{K}\left(v_{i}, v_{i}\right) \leq \frac{C}{h_{k}^{2}}\|v\|_{L_{r}^{2}(K)}^{2}
$$

for all $K$ and $i$ such that $v_{i}$ is nonzero on $K$. Here $a^{K}(\cdot, \cdot)$ denotes the restriction of the integrals appearing in $a(\cdot, \cdot)$ to $K$. We split the proof of (A.13) into several cases (wherein we omit the multilevel subscript $k$ for convenience).

We first consider elements $K$ which intersect $\Gamma_{0}$; i.e., $K=\left[r_{0}, r_{1}\right] \times\left[z_{0}, z_{1}\right]$ with $r_{0}=0$. The analysis proceeds by mapping to the unit square and using the 
equivalence of norms on a fixed finite dimensional subspace of bilinear functions defined on the unit square. For example, if $a=a_{r}$,

$$
\begin{aligned}
a^{K}\left(v_{i}, v_{i}\right) & =h \int_{0}^{1} \int_{0}^{1} \hat{r}\left(\left|\partial_{\hat{r}} \hat{v}_{i}\right|^{2}+\left|\partial_{\hat{z}} \hat{v}_{i}\right|^{2}\right) d \hat{r} d \hat{z} \\
& \leq C h\|\hat{v}\|_{L_{\hat{r}}^{2}\left((0,1)^{2}\right)}=C h^{-2}\|v\|_{L_{r}^{2}(K)} .
\end{aligned}
$$

Here $\hat{r}=r / h$ and $\hat{z}=\left(z-z_{0}\right) / h$. The above argument also works for $a=a_{\theta}$ with the bilinear approximation space $V_{h}^{\theta}$. It also works for the approximation space $\widetilde{V}_{h}^{\theta}$, because on the elements under consideration, the span of $r, 1 / r, r z$, and $z / r$ reduces to bilinear functions.

For $r_{0}>0$, bilinear elements with $a=a_{r}$ and $a=a_{\theta}$ are treated using the analogous well known results for bilinear finite elements in unweighted norms. For example, if $a=a_{r}$,

$$
\begin{aligned}
a^{K}\left(v_{i}, v_{i}\right) & \leq r_{1} \int_{K}\left(\left|\partial_{r} v_{i}\right|^{2}+\left|\partial_{z} v_{i}\right|^{2}\right) \mathrm{d} r \mathrm{~d} z \\
& \leq \frac{C r_{1}}{h^{2}}\|v\|_{L^{2}(K)}^{2} \leq \frac{C r_{1}}{r_{0} h^{2}}\|v\|_{L_{r}^{2}(K)}^{2} .
\end{aligned}
$$

The estimates for $a=a_{\theta}$ are similar.

Finally, we need to consider the case of $r_{0}>0$ and $\widetilde{V}_{h}^{\theta}$. We begin by observing that for any function $w$ in $\widetilde{V}_{h}^{\theta}$, the function $\left.q(r, z) \equiv r w(r, z)\right|_{K}$ is a linear combination of $r^{2}, 1, z r$, and $z$. Let $\hat{r}=\left(r-r_{0}\right) / h, \hat{z}=\left(z-z_{0}\right) / h$, and $\hat{q}(\hat{r}, \hat{z})=q(r, z)$. Let $\hat{V}$ denote the span of $\hat{r}^{2}, \hat{r}, \hat{r} \hat{z}, \hat{z}, 1$ on the unit square. Clearly we have $\hat{q} \in \hat{V}$. Because $\hat{V}$ is finite dimensional, scaling arguments show that there are constants $C_{1}$ and $C_{2}$ independent of $h$ and $q$ such that

$$
C_{1}\|q\|_{L^{2}(K)}^{2} \leq h^{2} \sum_{i=0}^{1} \sum_{j=0}^{2} q\left(r_{0}+j h / 2, z_{0}+i h\right)^{2} \leq C_{2}\|q\|_{L^{2}(K)}^{2} .
$$

Without loss of generality, let $x_{i}=\left(r_{0}, z_{1}\right)$ be the vertex corresponding to $v_{i}$. Then, letting $p(r, z) \equiv r v_{i}(r, z)$, we have by (A.14),

$$
\begin{aligned}
\left\|r v_{i}\right\|_{L^{2}(K)}^{2} & =\|p\|_{L^{2}(K)}^{2} \leq C h^{2} r_{0}^{2} v\left(r_{0}, z_{1}\right)^{2} \\
& \leq C h^{2} \sum_{i=0}^{1} \sum_{j=0}^{2}\left(r_{0}+j h / 2\right)^{2} v\left(r_{0}+j h / 2, z_{0}+i h\right)^{2} \\
& \leq C\|r v\|_{L^{2}(K)}^{2} .
\end{aligned}
$$

To prove (A.13), we use inverse inequalities for quadratic functions. Since $r v_{i}$ is quadratic on $K$,

$$
\begin{aligned}
a_{\theta}^{K}\left(v_{i}, v_{i}\right) & \leq \frac{1}{r_{0}} \int_{K}\left(\left|\partial_{r}\left(r v_{i}\right)\right|^{2}+\left|\partial_{z}\left(r v_{i}\right)\right|^{2}\right) \mathrm{d} r \mathrm{~d} z \\
& \leq \frac{1}{r_{0} h^{2}}\left\|r v_{i}\right\|_{L^{2}(K)}^{2} \leq \frac{C}{r_{0} h^{2}}\|r v\|_{L^{2}(K)}^{2} \\
& \leq \frac{C r_{1}}{r_{0} h^{2}}\|v\|_{L_{r}^{2}(K)}^{2} .
\end{aligned}
$$

This completes the proof of the lemma. 


\section{REFERENCES}

[1] C. Amrouche, C. Bernardi, M. Dauge, and V. Girault, Vector potentials in threedimensional non-smooth domains, Math. Methods Appl. Sci., 21 (1998), pp. 823-864. MR $1626990(99 \mathrm{e}: 35037)$

[2] F. Assous, P. Ciarlet, JR., and S. Labrunie, Theoretical tools to solve the axisymmetric Maxwell equations, Math. Methods Appl. Sci., 25 (2002), pp. 49-78. MR1874449 (2002j:78008)

[3] C. Bernardi, M. Dauge, and Y. Maday, Spectral methods for axisymmetric domains, vol. 3 of Series in Applied Mathematics (Paris), Gauthier-Villars, Éditions Scientifiques et Médicales Elsevier, Paris, 1999. Numerical algorithms and tests due to Mejdi Azaïez. MR1693480 (2000h:65002)

[4] S. Beuchler, Fast solvers for degenerated problems, Tech. Rep. SFB393-Preprint 4, Technische Universität Chemnitz, SFB 393 (Germany), 2003.

[5] S. Börm And R. Hiptmair, Analysis of tensor product multigrid, Numer. Algorithms, 26 (2001), pp. 219-234. MR 1832541 (2002a:65165)

[6] - Multigrid computation of axisymmetric electromagnetic fields, Adv. Comput. Math., 16 (2002), pp. 331-356. MR 1894928 (2003d:78042)

[7] J. H. Bramble And X. ZHANG, The analysis of multigrid methods, in Handbook of numerical analysis, Vol. VII, North-Holland, Amsterdam, 2000, pp. 173-415. MR:1804746 (2001m:65183)

[8] - Uniform convergence of the multigrid $V$-cycle for an anisotropic problem, Math. Comp., 70 (2001), pp. 453-470. MR1709148 (2001g:65134)

[9] A. BRandt, Multigrid techniques: 1984 guide with applications to fluid dynamics, vol. 85 of GMD-Studien, Gesellschaft für Mathematik und Datenverarbeitung mbH, St. Augustin, 1984. MR0772748 (87c:65139b)

[10] W. HackBusch, Multi-Grid Methods and Applications, no. 4 in Springer series in Computational Mathematics, Springer-Verlag, Berlin, 1985. MR0814495 (87e:65082)

[11] G. H. Hardy, J. E. Littlewood, and G. Pólya, Inequalities, Cambridge Mathematical Library, Cambridge University Press, Cambridge, 1988. Reprint of the 1952 edition. MR0944909 (89d:26016)

[12] H. HochstadT, The functions of mathematical physics, vol. XXIII of Pure and Applied Mathematics, Wiley-Interscience, New York, 1971. MR0499342 (58:17241)

[13] A. Kufner, Weighted Sobolev spaces, A Wiley-Interscience Publication, John Wiley \& Sons Inc., New York, 1985. Translated from the Czech. MR0802206 (86m:46033)

[14] N. Neuss, $V$-cycle convergence with unsymmetric smoothers and application to an anisotropic model problem, SIAM J. Numer. Anal., 35 (1998), pp 1201-1212. MR.1619887 (99d:65109)

[15] A. Reusken, On a robust multigrid solver, Computing, 56 (1996), pp. 303-322. International GAMM-Workshop on Multi-level Methods (Meisdorf, 1994). MR.1393011 (97g:65260)

[16] G. N. Watson, A Treatise on the Theory of Bessel Functions, Cambridge University Press, Cambridge, 2nd ed., 1952. MR1349110 (96i:33010)

[17] H. Yserentant, The convergence of multi-level methods for solving finite-element equations in the presence of singularities, Math. Comp., 47 (1986), pp. 399-409. MR0856693 (88d:65149)

Department of Mathematics, University of Florida, Gainesville, Florida 32611-8105

E-mail address: jayg@math.ufl.edu

Department of Mathematics, Texas A\&M University, College Station, Texas 778433368

E-mail address: pasciak@math.tamu.edu 